\title{
Structure and properties of the stable two-dimensional conducting polymer $\mathrm{Mg}_{5} \mathrm{C}_{60}$
}

\author{
D. Quintavalle, ${ }^{1, *}$ F. Borondics, ${ }^{2, \dagger}$ G. Klupp, ${ }^{2}$ A. Baserga, ${ }^{3}$ F. Simon, ${ }^{1}$ A. Jánossy, ${ }^{1}$ K. Kamarás, ${ }^{2}$ and S. Pekker ${ }^{2}$ \\ ${ }^{1}$ Budapest University of Technology and Economics, Institute of Physics and Condensed Matter Physics, \\ Research Group of the Hungarian Academy of Sciences, P.O. Box 91, Budapest H-1521, Hungary \\ ${ }^{2}$ Research Institute for Solid State Physics and Optics, Hungarian Academy of Sciences, P.O. Box 49, Budapest H-1525, Hungary \\ ${ }^{3}$ Center for Nanoengineered Materials and Surfaces (NEMAS), Dipartimento di Ingegneria Nucleare, \\ Politecnico di Milano, I-20133 Milano, Italy
}

(Received 25 January 2008; revised manuscript received 14 March 2008; published 18 April 2008)

\begin{abstract}
We present a study on the structural, spectroscopic, conducting, and magnetic properties of $\mathrm{Mg}_{5} \mathrm{C}_{60}$, which is a two-dimensional (2D) fulleride polymer. The polymer phase is stable up to the exceptionally high temperature of $823 \mathrm{~K}$. The infrared and Raman studies suggest the formation of single bonds between the fulleride ions and possibly $\mathrm{Mg}-\mathrm{C}_{60}$ covalent bonds. $\mathrm{Mg}_{5} \mathrm{C}_{60}$ is a metal at ambient temperature, as shown by electron spin resonance and microwave conductivity measurements. The smooth transition from a metallic to a paramagnetic insulator state below $200 \mathrm{~K}$ is attributed to Anderson localization driven by structural disorder.
\end{abstract}

DOI: 10.1103/PhysRevB.77.155431

PACS number(s): 61.48.-c, 76.30.Pk, 78.30.-j

\section{INTRODUCTION}

The unusual physical and structural properties of alkali metal intercalated fulleride polymers have received considerable attention since the discovery in 1994 of the linear $A \mathrm{C}_{60}(A=\mathrm{K}, \mathrm{Rb}, \mathrm{Cs})$ conducting polymers. ${ }^{1}$ Chemical reactions between charged fulleride ions in solids are rather common. The polymer phases usually form spontaneously or under a mild pressure from the monomeric crystalline salts. Polymers of $\mathrm{C}_{60}^{n-}$ anions are usually unstable above temperatures of about $400 \mathrm{~K}$. The depolymerization is reversible, in contrast to photopolymerization ${ }^{2}$ and pressure polymerization of neutral $\mathrm{C}_{60} 0^{3,4}$ According to quantum chemical calculations, ${ }^{5}[2+2]$ cycloaddition is favored in $A_{n} \mathrm{C}_{60}$ for low values of $n$, and configurations with single interfullerene bonds are more stable for $n \geqslant 3$. In agreement with these expectations, the stable form of $A C_{60}^{-}$is a cycloaddition polymer, ${ }^{6}$ while single intermolecular bonds occur between $\mathrm{C}_{60}^{n-}$ anions in $\mathrm{Na}_{4} \mathrm{C}_{60}$ (Ref. 7) and $\mathrm{Na}_{2} \mathrm{RbC}_{60} \cdot{ }^{8}$ Single and double bonds can simultaneously appear, as has been recently demonstrated in $\mathrm{Li}_{4} \mathrm{C}_{60},{ }^{9}, 10$ wherein single bonds connect polyfulleride chains held together by $[2+2]$ cycloaddition bonds.

Fulleride polymers have small electronic bandwidths and large on-site electron repulsion and are thus strongly thus correlated electron systems. Small differences in the lattice parameters and/or variations in the chain orientation ${ }^{11}$ can profoundly change the ground state. For example, the linear polymer $\mathrm{KC}_{60}$ has a metallic ground state while $\mathrm{RbC}_{60}$ and $\mathrm{CsC}_{60}$ with the same type of polymer chains but different chain orientations undergo a metal-insulator transition to an antiferromagnetic spin density wave ground state. $^{12}$ Electron-electron correlations play an important role in twodimensional (2D) fulleride polymers as well: $\mathrm{Na}_{4} \mathrm{C}_{60}$ is a strongly correlated metal, ${ }^{7}$ while $\mathrm{Li}_{4} \mathrm{C}_{60}$ is a nonmagnetic insulator. ${ }^{10}$

In this paper, we present a study of the structure and physical properties of the recently synthesized fulleride polymer $\mathrm{Mg}_{5} \mathrm{C}_{60}$. In a previous study of $\mathrm{Mg}_{x} \mathrm{C}_{60}$, a stoichiometric compound ${ }^{13}$ was reported for $x=4$. In the present work, we improved the synthesis and conclude from a series of samples with varying $\mathrm{Mg}$ content that the homogeneous phase lies in a range of $\mathrm{Mg}$ concentrations between $x=5$ and $x=5.5 . \mathrm{Mg}_{5} \mathrm{C}_{60}$ is the only known example of an alkaline earth fulleride polymer. The polymer phase was found to be stable up to the remarkably high temperature of $823 \mathrm{~K}$ by electron spin resonance. Other fullerene polymers are stable to much lower temperatures and depolymerize at about $400-500 \mathrm{~K}$. We find that $\mathrm{Mg}_{5} \mathrm{C}_{60}$ is a $2 \mathrm{D}$ polymer that is metallic at a high temperature and undergoes a gradual transition to an insulating ground state as the temperature is lowered.

Previous studies of alkaline earth fullerides mostly focused on $\mathrm{Ca}_{x} \mathrm{C}_{60}$ and $\mathrm{Ba}_{x} \mathrm{C}_{60}$ (Refs. 14 and 15) superconductors, which are not polymers. An early study of $\mathrm{Mg}$ doped fulleride films reported an insulating behavior for all $\mathrm{Mg}$ concentrations, ${ }^{16}$ which is in disagreement with our present results.

\section{EXPERIMENT}

Samples of $\mathrm{Mg}_{x} \mathrm{C}_{60}$ with nominal concentrations $x=4-6$, which are in steps of $\Delta x=0.5 \pm 0.05$, were prepared by solid state reaction between $\mathrm{C}_{60}$ and pure $\mathrm{Mg}$ powder under argon atmosphere in a dry box. The powder mixture was placed in carbon steel containers to avoid reaction between the $\mathrm{Mg}$ grains and quartz vessels. $\mathrm{Mg}$ grain surface activation at $753 \mathrm{~K}$ was followed by several annealing steps at temperatures from 653 to $723 \mathrm{~K}$. To improve the homogeneity of the sample, we used $\mathrm{Mg}$ powder with smaller grain sizes than in the previous preparation. ${ }^{13}$ Powders were reground before each annealing step.

X-ray powder diffraction was performed using a HUBER G670 Guinier image plate camera in transmission mode and highly monochromatic $\mathrm{Cu} K \alpha_{1}$ radiation. Infrared transmission spectra were recorded on pressed $\mathrm{KBr}$ pellets with a Bruker IFS 28 Fourier transform infrared spectrometer under dynamic vacuum. Raman spectra were collected using a T64000 Jobin-Yvon spectrometer in triple grating configura- 
tion with spectral resolution better than $3 \mathrm{~cm}^{-1}$. The $532 \mathrm{~nm}$ line of a frequency-doubled neodymium doped yttrium aluminum garnet laser, Nd-YAG, was the excitation source. The diameter of the spot was $100 \mu \mathrm{m}$ corresponding to a nominal irradiance of about $150 \mathrm{~W} / \mathrm{cm}^{2}$.

Microwave conductivity was measured at $10 \mathrm{GHz}$ on fine $\mathrm{Mg}_{5} \mathrm{C}_{60}$ powders mixed with high purity $\mathrm{SnO}_{2}$ powder to electrically isolate the grains. A $10 \mathrm{GHz}$ copper cylindrical TE011 resonant cavity was used for the cavity perturbation technique conductance measurements. ${ }^{17,18}$ In this method, changes in the quality factor are measured using lock-in detection of the frequency modulated response. The sample is placed at the cavity center where the microwave magnetic field is maximum and microwave currents encircle the grains. This geometry is well adapted for fine powders because the microwave electric field is not shielded by depolarization effects. ${ }^{18}$ The typical grain size was much smaller than the microwave penetration depth, as confirmed by the symmetric Lorentzian electron spin resonance (ESR) line shape measured at the same frequency. The temperature dependence of the conductivity $\sigma(T)$, is proportional to $1 / Q-1 / Q_{0} . Q$ and $Q_{0}$, which are the quality factors of the cavity with and without the sample, respectively, were measured in two separate runs. $Q_{0}$ was about 16000 and it changed little with temperature. Ohmic losses of a single spherical grain with radius $r$ and conductivity $\sigma$ are proportional to $r^{5} \sigma$. The determination of the absolute value of the conductivity is not possible without a detailed knowledge of the distribution of $r$.

ESR spectroscopy was performed at 9 and $225 \mathrm{GHz}$ on samples sealed in quartz tubes under 200 mbar He pressure. At $9 \mathrm{GHz}$, a commercial Bruker ELEXSYS 500 spectrometer was used, the $225 \mathrm{GHz}$ spectra were recorded by a homebuilt spectrometer. The spin susceptibility was obtained by integrating the ESR spectra. $\mathrm{MgO}$ doped with $1.5 \mathrm{ppm}$ of $\mathrm{Mn}^{2+}$ ions was the ESR intensity reference to obtain the susceptibility between 100 and $300 \mathrm{~K}$. Below $100 \mathrm{~K}$, the $\mathrm{Mn}: \mathrm{MgO}$ ESR saturates and fine orthorhombic polymeric $\mathrm{KC}_{60}$ powder (a low conductivity metal with a temperature independent susceptibility) was used as an intensity reference.

\section{RESULTS AND DISCUSSION}

\section{A. X-ray diffraction}

Powder diffractograms of a series of $\operatorname{Mg}_{x} \mathrm{C}_{60}$ salts (Fig. 1) show that the samples are single phase for nominal concentration values between 5 and 5.5. The peak at about $36.3^{\circ}$ demonstrates that above a nominal composition of $x=5.5$, some $\mathrm{Mg}$ metal is present. The $\mathrm{x}$-ray results also indicate that $\mathrm{Mg}_{5} \mathrm{C}_{60}$ is not a line phase, the structure presented here and in Ref. 13 comprises a range of stoichiometries between $x=5$ and 5.5. In the previous paper, the single phase material has been obtained for a nominal composition of $x=4$ and has been indexed as rombohedral with lattice parameters $a=b=9.22 \AA, c=25.25 \AA, \gamma=120^{\circ}$. These values indicate the formation of two-dimensional hexagonal $\mathrm{C}_{60}$ polymeric sheets in the $a b$ plane. The material belongs to the $R \overline{3} \mathrm{~m}$

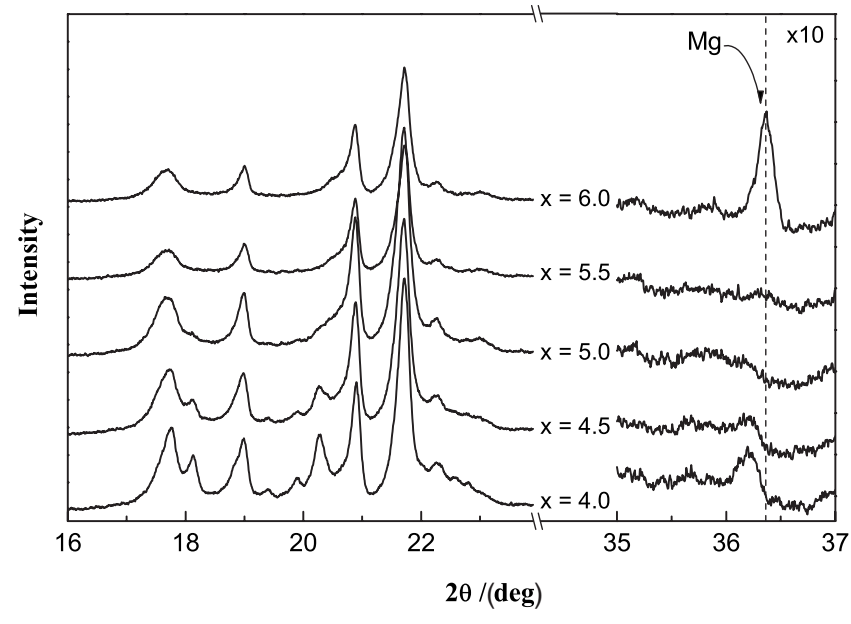

FIG. 1. X-ray powder diffraction data for a series of $\operatorname{Mg}_{x} \mathrm{C}_{60}$ polymers, with nominal concentrations $x$ varying from 4.0 to 6.0. The material is single phase for $x=5$ and 5.5. The dotted line above $36^{\circ}$ corresponds to $\mathrm{Mg}$ metal.

space group. $\mathrm{C}_{60}$ molecules are positioned at the fractional coordinates $(0,0,0),(2 / 3,1 / 3,1 / 3)$, and $(1 / 3,2 / 3,2 / 3)$, while the $\mathrm{Mg}$ positions are $(0,0,0.23)$ and $(0,0,0.43)$. The improved synthesis method using fine-powdered magnesium metal resulted in a more reliable magnesium content than the previous one. However, even in the better defined material, we can determine the positions of only four of the five magnesium ions. A range of alkali concentrations without any change in the structure of the polymer has been observed in $\mathrm{Li}_{x} \mathrm{C}_{60}(3 \leqslant x \leqslant 5),{ }^{10}$ with the structural parameters given by a Rietveld fit corresponding to $x=4$. Subsequent photoemission data on $\mathrm{Li}_{x} \mathrm{C}_{60}$ thin films with the same structure showed an incomplete charge transfer, which do not change throughout the homogeneity range. ${ }^{19}$

\section{B. Infrared and Raman spectroscopy}

The infrared transmission and Raman scattering spectra of $\mathrm{Mg}_{5} \mathrm{C}_{60}$ are shown in Fig. 2. The low-frequency part corre-

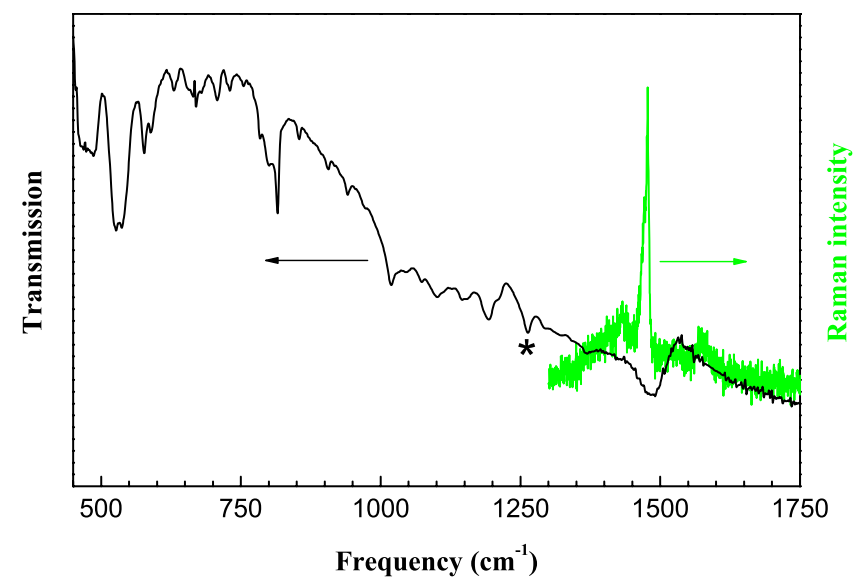

FIG. 2. (Color online) Infrared transmission and Raman scattering spectra of $\mathrm{Mg}_{5} \mathrm{C}_{60}$. The feature at $1480 \mathrm{~cm}^{-1}$, which is visible in both spectra, is assigned to the $A_{g}(2)$ vibrational mode of $\mathrm{C}_{60}$. The infrared line marked with an asterisk is an extrinsic impurity mode. 


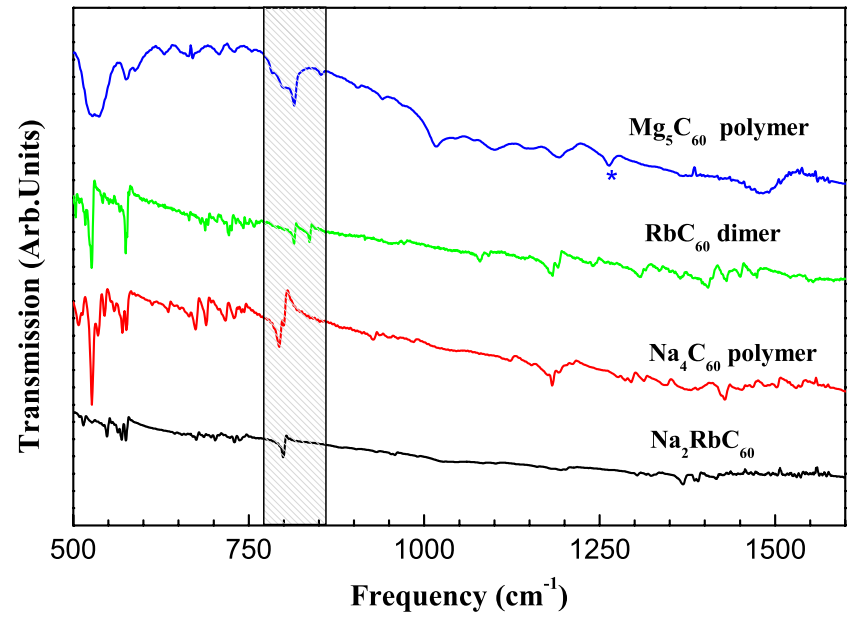

FIG. 3. (Color online) Comparison of the infrared spectra of various singly bonded fulleride oligo- and polymers. All the spectra show an absorption line around $815 \mathrm{~cm}^{-1}$ (indicated by the bar), which is attributed to single bonds between $\mathrm{C}_{60}$ molecules. The infrared line marked with an asterisk is an extrinsic impurity mode. The curves are shifted along the vertical axis for clarity.

sponds to a symmetry-reduced $\mathrm{C}_{60}$ molecule with the principal $T_{1 u}$ modes at 523,576 , and $1193 \mathrm{~cm}^{-1}$ and several new modes, especially in the region between 700 and $900 \mathrm{~cm}^{-1}$. Such marked changes in the spectrum indicate a symmetry lowering to an extent that is usually caused by intermolecular bonds (i.e., polymerization) ${ }^{22}$ and not just deformation of isolated $\mathrm{C}_{60}$ balls in a crystal. The line around $815 \mathrm{~cm}^{-1}$ is of particular interest. This line appears in the spectrum of all $\mathrm{C}_{60}$-based polymers, ${ }^{20,21}$ wherein the $\mathrm{C}_{60}$ molecules are connected by single bonds (see Fig. 3). We consider the appearance of this characteristic peak in $\mathrm{Mg}_{5} \mathrm{C}_{60}$ as proof for single bonds between fulleride ions.

The high-frequency region around the $T_{1 u}(4)$ mode shows an unusual pattern, which is dominated by a broad feature around $1480 \mathrm{~cm}^{-1}$. This feature cannot be explained by a shift in the $T_{1 u}(4)$ mode by charge transfer from the $\mathrm{Mg}$ atom nor by polymerization effects, which are both known to reduce the vibrational frequencies. ${ }^{22,23}$ Instead, we assign this peak to the $A_{g}(2)$ vibrational mode, which is Raman active in undistorted $\mathrm{C}_{60}$. Our assignment is supported by the fact that the peak is also dominant in the Raman spectrum. A similar effect was reported for $\mathrm{C}_{60}$ monolayers adsorbed on metal ${ }^{24}$ and semiconductor surfaces. ${ }^{25}$ In those systems, an intense infrared absorption above the highest-frequency $T_{1 u}$ mode is observed, together with a Raman counterpart, ${ }^{26}$ which is attributed to the $A_{g}(2)$ mode rendered infrared active by symmetry reduction and amplified by the "vibrational phase relaxation" mechanism. ${ }^{27}$ Prerequisites for this mechanism are (1) a free-electron continuum and (2) low-frequency vibrational or translational modes, which mediate the coupling. According to our ESR and microwave data (see below), conducting electrons are present in $\mathrm{Mg}_{5} \mathrm{C}_{60}$ and it is known from Raman spectroscopy of $\mathrm{C}_{60}$ photopolymers that intermolecular vibrations appear around $100 \mathrm{~cm}^{-1}$ (Ref. 2), thus, both criteria are met. In the case of $\mathrm{Mg}_{5} \mathrm{C}_{60}$ shown in Fig. 2, the mode is both infrared and Raman active but, unlike in Refs. 24 and 25 , in both cases shifted up by $12 \mathrm{~cm}^{-1}$ compared to

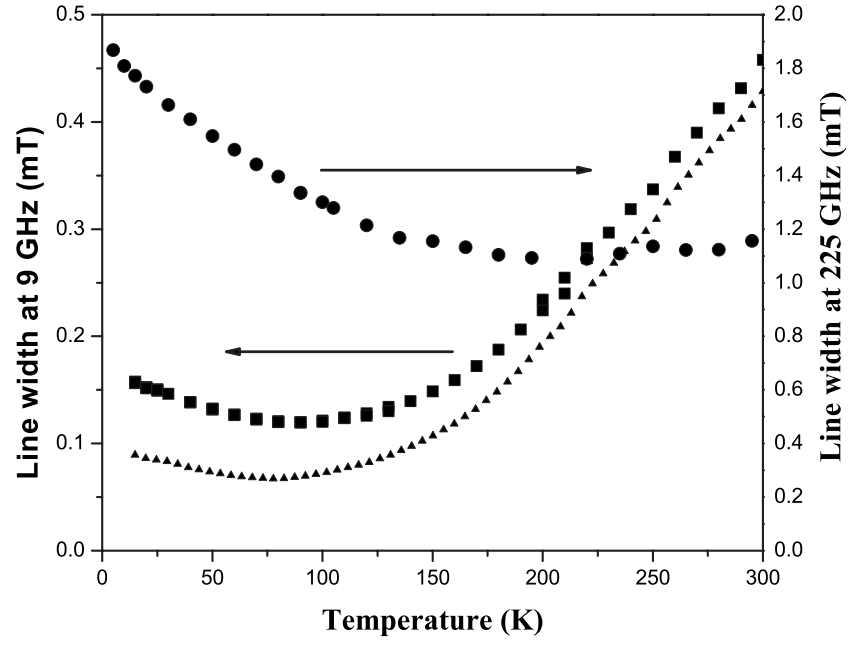

FIG. 4. Temperature dependence of the ESR linewidth of $\mathrm{Mg}_{5} \mathrm{C}_{60}$ at $9 \mathrm{GHz}$ (squares) and $225 \mathrm{GHz}$ (circles). The temperature dependence of the frequency independent component of the linewidth $a(T)$ is also reported (triangles). For temperatures above $175 \mathrm{~K}$, the linewidth at $9 \mathrm{GHz}$ linearly increases with temperature, as is expected in metals in which the main relaxation mechanism is the electron-phonon interaction.

the $1468 \mathrm{~cm}^{-1}$ pristine $A_{g}(2)$ mode. Such blueshift, although smaller, has been observed in $\mathrm{C}_{60}$ monolayers adsorbed on platinum, ${ }^{28}$ wherein it was explained by covalent bonding to the metal, resulting in electron back transfer from the fullerene to the metal surface. Covalent bonding to at least some of the $\mathrm{Mg}$ ions cannot be excluded in the present case either considering the close values of the $\mathrm{Mg}$ ionization potential and the lowest unoccupied molecular orbital level of $\mathrm{C}_{60}$. The phenomenon described here warrants further investigation since it was not seen in bulk fulleride salts before.

\section{Microwave conductivity and electron spin resonance}

In the following, we discuss the microwave conductivity and ESR measurements, which show that $\mathrm{Mg}_{5} \mathrm{C}_{60}$ is metallic above room temperature and becomes insulating at low temperatures.

The ESR spectrum consists of a single Lorentzian line at all temperatures both at 9 and $225 \mathrm{GHz}$, confirming the phase purity of the sample. Phase inhomogeneities usually split the line or at least inhomogeneously broaden it due to differences in the $g$ factor. If the $\mathrm{Mg}$ concentration were inhomogeneous, then the high-frequency ESR lines would be distorted especially in the low temperature insulating state where spatial variations are not averaged by spin diffusion.

In Fig. 4, we show the temperature dependence of the ESR linewidth. The $9 \mathrm{GHz}$ line is a symmetric Lorentzian with a $0.45 \mathrm{mT}$ linewidth at room temperature. The line broadens with increasing frequency. The frequency and the temperature dependences of the linewidth may be separated to give $\Delta H=a(T)+b(T) H$. The frequency independent term, $a(T)$, is almost constant below $100 \mathrm{~K}$ (see Fig. 4) and linearly increases with temperature as in metals, in which the main relaxation mechanism is the electron-phonon interaction. $^{31}$ The term $b(T)$ increases with frequency and 


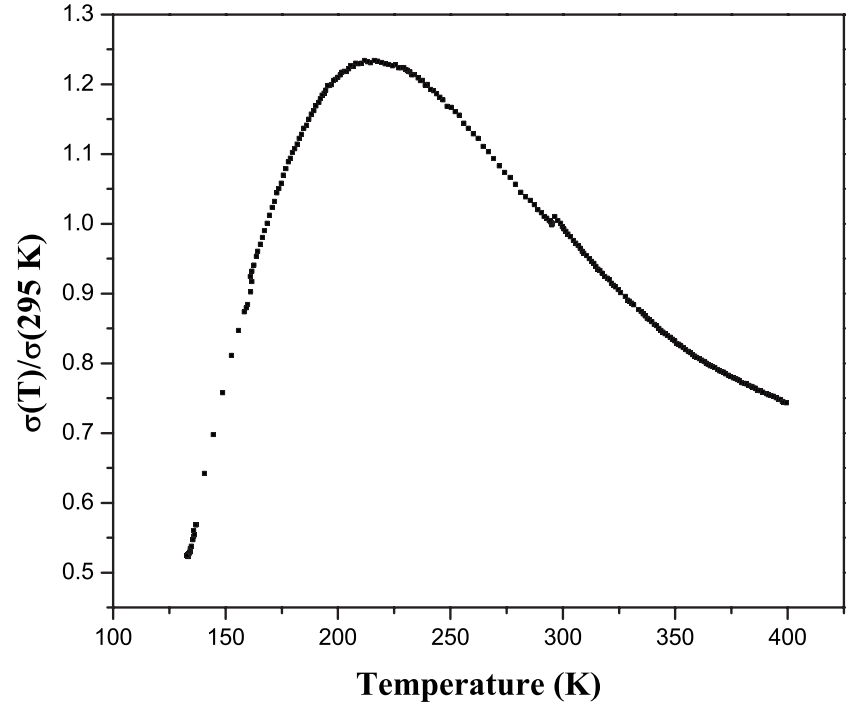

FIG. 5. Microwave conductivity at $10 \mathrm{GHz}$ of $\mathrm{Mg}_{5} \mathrm{C}_{60}$. The conductivity has a smooth transition from a metal to an insulator behavior at about $200 \mathrm{~K}$, which we attribute to Anderson localization of the electronic states.

with lowering of the temperature. The line broadening at $225 \mathrm{GHz}$ is due to a partially averaged $g$-factor anisotropy. We attribute the broadening at $T<200 \mathrm{~K}$ (see Fig. 4) to the inhomogeneous interaction between paramagnetic defects randomly distributed in the lattice.

Figure 5 displays the temperature dependence of the microwave conductivity of $\mathrm{Mg}_{5} \mathrm{C}_{60}$ normalized to $295 \mathrm{~K}$. At high temperatures, the conductivity decreases with increasing temperature, as is usual in metals wherein scattering is due to phonons. The low temperature behavior is different from that of usual metals. The conductivity has a broad maximum and decreases below $200 \mathrm{~K}$. This behavior corresponds to a smooth transition between metallic and insulating states.

A deeper insight into the nature of this transition is given by the temperature dependence of the spin susceptibility shown in Fig. 6. For $T \geqslant 200 \mathrm{~K}$, the spin susceptibility has a value of $2 \times 10^{-5} \mathrm{emu} / \mathrm{mole}$ and is almost independent of temperature, as expected for the Pauli susceptibility of a degenerate electron gas. In normal metals, the spin susceptibility is related to the density of states at the Fermi level $n\left(E_{F}\right)$ : $\chi=1 / 4 g^{2} \mu_{B}^{2} n\left(E_{F}\right)$, where $g$ is the spectroscopic $g$ factor and $\mu_{B}$ is the Bohr magneton. At lower temperatures, a Curie contribution characteristic of a small concentration of localized spins dominates the ESR spectrum. The change of the temperature dependence of the static susceptibility from Pauli to Curie-like is gradual and there is no well-defined phase transition. The presence of a gap in the electron energy spectrum can be excluded because the spin susceptibility does not disappear even at the lowest temperatures. We do not observe a Mott-Hubbard transition, which usually has a well-defined transition temperature and gives rise to a magnetically ordered ground state. We suggest instead that this smooth transition to an insulating ground state is due to Anderson localization. At a certain degree of disorder of the lattice, the electronic wave functions with an energy below

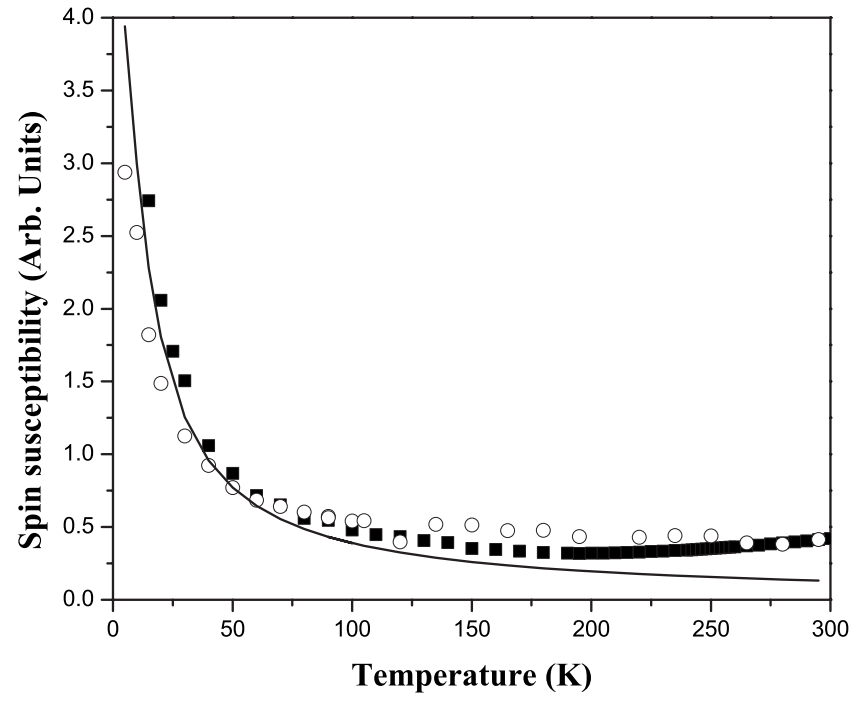

FIG. 6. Temperature dependence of the spin susceptibility of $\mathrm{Mg}_{5} \mathrm{C}_{60} .225 \mathrm{GHz}$ ESR measurements (empty circles) are normalized to the $9 \mathrm{GHz}$ ones (squares) at $300 \mathrm{~K}$. The continuous line is the Brillouin function of spin $1 / 2$ in $8.1 \mathrm{~T}$ field normalized at $50 \mathrm{~K}$ to the $225 \mathrm{GHz}$ ESR measurement. At all the frequencies, the spin susceptibility shows a broad transition from a Pauli- to a Brillouinlike temperature dependence, suggesting a localization of the electronic states. The susceptibility does not disappear at the lowest temperatures, excluding a gap in the excitation spectrum.

the mobility edge, $E_{c}$, become localized. ${ }^{29,30}$ If the Fermi energy lies below $E_{c}$, the ground state is insulating. On the other hand, electrons become delocalized and the behavior is metallic when the temperature is much higher than $E_{c} / k_{B}$. The temperature dependence of the conductivity of $\mathrm{Mg}_{5} \mathrm{C}_{60}$ is dominated by the thermal activation of the electrons above the mobility edge at low temperatures and by the electronphonon relaxation for $T>200 \mathrm{~K}$. The description of the behavior near $200 \mathrm{~K}$, where the two contributions are comparable, requires a deeper knowledge of the disorder potential and of the phonon structure.

We measured a lower limit of the depolymerization temperature by searching for changes in the ESR spectra after annealing at higher temperatures. The sealed $\mathrm{Mg}_{5} \mathrm{C}_{60}$ sample was heated at a rate of $10 \mathrm{~K} / \mathrm{min}$ to each temperature, kept there for $15 \mathrm{~min}$, and quenched into water and then the $9 \mathrm{GHz}$ ESR spectra were recorded at ambient temperature. We repeated this cycle twice in $25 \mathrm{~K}$ steps between 300 and $848 \mathrm{~K}$. We found that treatment up to $823 \mathrm{~K}$, leaves the ESR spectrum unchanged. At $848 \mathrm{~K}$, the ESR spectrum and the color of the quartz sample holder changed. These changes indicate reaction of $\mathrm{Mg}$ (diffusing out of the sample) with quartz. It is not clear whether without this reaction depolymerization would take place. We conclude that the polymer phase is stable for at least $30 \mathrm{~min}$ at $823 \mathrm{~K}$.

\section{CONCLUSION}

$\mathrm{Mg}_{x} \mathrm{C}_{60}$ with $x=5-5.5$ is a single phase, two-dimensional fulleride polymer. X-ray diffraction indicates a finite 
stoichiometry range with the same structure. Vibrational spectroscopy reveals that the $\mathrm{Mg}$-fullerene interaction is more complicated than a simple charge transfer with a possible covalent component. Microwave conductivity shows that $\mathrm{Mg}_{5} \mathrm{C}_{60}$ is metallic for $T>200 \mathrm{~K}$ and this is confirmed by the temperature dependence of the spin susceptibility and of the ESR linewidth. We attribute the transition from metallic to paramagnetic insulating states below $200 \mathrm{~K}$ to a disorder driven Anderson localization. The stability of the polymeric structure of $\mathrm{Mg}_{5} \mathrm{C}_{60}$ up to temperatures above $800 \mathrm{~K}$ is exceptional, all other polymers of charged fullerides con- nected by single or double C-C bonds decompose at much lower temperature.

\section{ACKNOWLEDGMENTS}

We gratefully acknowledge G. Oszlányi for helpful discussions and L. Forró for providing the $X$-band ESR spectrometer at the EPFL. This work was supported by the Hungarian National Research Fund (OTKA) through Grants No. T 049338, No. T 046700, No. TS049881, No. F61733, and No. NK60984.
*Corresponding author: dario@esr.phy.bme.hu

${ }^{\dagger}$ Present address: Advanced Light Source Division, Lawrence Berkeley National Laboratory, Berkeley, CA 94720-8226.

${ }^{1}$ S. Pekker, L. Forró, L. Mihály, and A. Jánossy, Solid State Commun. 90, 349 (1994).

${ }^{2}$ M. Rao, P. Zhou, K. A. Wang, G. T. Hager, J. M. Holden, Y. Wang, W. T. Lee, X. X. Bi, and P. C. Eklund, Science 259, 955 (1993).

${ }^{3}$ Y. Iwasa et al., Science 264, 1570 (1994).

${ }^{4}$ M. Nunez-Regueiro, L. Marques, J.-L. Hodeau, O. Béthoux, and M. Perroux, Phys. Rev. Lett. 74, 278 (1995).

${ }^{5}$ S. Pekker and G. Oszlányi, Synth. Met. 103, 2411 (1999).

${ }^{6}$ P. W. Stephens, G. Bortel, G. F. L., M. Tegze, A. Jánossy, S. Pekker, G. Oszlányi, and L. Forró, Nature (London) 370, 636 (1994).

${ }^{7}$ G. Oszlányi, G. Baumgartner, G. Faigel, and L. Forró, Phys. Rev. Lett. 78, 4438 (1997).

${ }^{8}$ G. M. Bendele, P. W. Stephens, K. Prassides, K. Vavekis, K. Kordatos, and K. Tanigaki, Phys. Rev. Lett. 80, 736 (1998).

${ }^{9}$ S. Margadonna, D. Pontiroli, M. Belli, T. Shiroka, M. Ricco, and M. Brunelli, J. Am. Chem. Soc. 126, 15032 (2004).

${ }^{10}$ M. Riccó, T. Shiroka, M. Belli, D. Pontiroli, M. Pagliari, G. Ruani, D. Palles, S. Margadonna, and M. Tomaselli, Phys. Rev. B 72, 155437 (2005).

${ }^{11}$ P. Launois, R. Moret, J. Hone, and A. Zettl, Phys. Rev. Lett. 81, 4420 (1998).

${ }^{12}$ F. Bommeli, L. Degiorgi, P. Wachter, Ö. Legeza, A. Jánossy, G. Oszlanyi, O. Chauvet, and L. Forro, Phys. Rev. B 51, 14794 (1995)

${ }^{13}$ F. Borondics, G. Oszlányi, G. Faigel, and S. Pekker, Solid State Commun. 127, 311 (2003).

${ }^{14}$ A. R. Kortan, N. Kopylov, S. Glarum, E. M. Gyorgy, A. P. Ramirez, R. M. Fleming, F. A. Thiel, and R. C. Haddon, Nature
(London) 355, 529 (1992).

${ }^{15}$ M. Baenitz, M. Heinze, K. Luders, H. Werner, R. Schlogl, M. Weiden, G. Sparn, and F. Steglich, Solid State Commun. 96, 539 (1995).

${ }^{16}$ Y. Chen, F. Stepniak, J. H. Weaver, L. P. F. Chibante, and R. E. Smalley, Phys. Rev. B 45, 8845 (1992).

${ }^{17}$ B. Nebendahl, D.-N. Peligrad, M. Požek, A. Dulčić, and M. Mehring, Rev. Sci. Instrum. 72, 1876 (2001).

${ }^{18}$ L. I. Buravov and I. F. Shchegolev, Instrum. Exp. Tech. 14, 528 (1971).

${ }^{19}$ R. Macovez, R. Savage, L. Venema, F. Hennies, J. Schiessling, K. Kamarás, and P. Rudolf, J. Phys. Chem. C 112, 2998 (2008).

${ }^{20}$ N. Tagmatarchis, H. Shinohara, T. Pichler, M. Krause, and H. Kuzmany, J. Chem. Soc., Perkin Trans. 2 2000, 2361.

${ }^{21}$ G. Klupp, F. Borondics, G. Oszlányi, and K. Kamarás, AIP Conf. Proc. 685, 62 (2003).

${ }^{22}$ A. M. Rao, P. C. Eklund, J.-L. Hodeau, L. Marques, and M. Núñez-Regueiro, Phys. Rev. B 55, 4766 (1997).

${ }^{23}$ T. Wågberg and B. Sundqvist, Phys. Rev. B 65, 155421 (2002).

${ }^{24}$ P. Rudolf, R. Raval, P. Dumas, and G. P. Williams, Appl. Phys. A: Mater. Sci. Process. 75, 147 (2002).

${ }^{25}$ P. Dumas, M. Gruyters, P. Rudolf, Y. He, L.-M. Yu, G. Gensterblum, R. Caudano, and Y. J. Chabal, Surf. Sci. 368, 330 (1996).

${ }^{26}$ A. Peremans, Y. Caudano, P. A. Thiry, P. Dumas, W. Q. Zheng, A. Le Rille, and A. Tadjeddine, Phys. Rev. Lett. 78, 2999 (1997).

${ }^{27}$ W. Erley and B. N. J. Persson, Surf. Sci. 218, 494 (1989).

${ }^{28}$ C. Cepek, A. Goldoni, and S. Modesti, Phys. Rev. B 53, 7466 (1996).

${ }^{29}$ P. W. Anderson, Phys. Rev. 109, 1492 (1958).

${ }^{30}$ N. F. Mott, Adv. Phys. 16, 49 (1967).

${ }^{31}$ Y. Yafet, Solid State Phys. 14, 1 (1963). 